\title{
A practical prediction method using an elementary response wave form for the road traffic noise at a T-type intersection
}

\author{
Mitsuo Ohta and Yasuo Mitani \\ Faculty of Engineering, Hiroshima University, \\ Shitami, Saijo-cho, Higashi-Hiroshima, 724 Japan
}

(Received 24 November 1988)

\begin{abstract}
A general method of predicting a road traffic noise generated from an arbitrary nonPoisson type traffic flow was previously proposed for practical use, based on the information on an elementary response wave form when one vehicle passes directly in front of an observation point. By developing this prediction method, in this report, more advanced investigation applicable to a fairly complicated acoustic environment is described by applying it to the actual road traffic noise observed especially at a T-type road intersection in a city area. At this time, the effects of the surrounding background noises are reasonably taken into account for predicting the road traffic noise.
\end{abstract}

PACS number: 43. 60. $\mathrm{Cg}$, 43. 50. $\mathrm{Yw}$

\section{INTRODUCTION}

As is well-known, for the purpose of predicting a road traffic noise, it is essentially important to grasp the concrete characteristics of the traffic flow and the sound propagation. In the actual traffic flow, an arbitrary non-Poisson type traffic flow distribution can be often seen rather than in the form of an idealized Poisson type, since it is seldom that the traffic conditions are ideal. On the other hand, for obtaining quantitatively the sound propagation characteristic in a complicated acoustic environment, it is very convenient and practical to first utilize the information on an elementary response wave form of road traffic noise on a decibel scale as the time pattern observed when one vehicle passes directly in front of an observation point. From this fundamental viewpoint, in the previous work, ${ }^{1)}$ a general method of predicting a road traffic noise generated from an arbitrary traffic flow was proposed for practical use, based on the information on this elementary response wave form. This prediction method was shown to be in a unified but simplified prediction form with the flexible applica- bility to the arbitrary types of vehicles situation and sound propagation environment. However, the validity of the previous work was experimentally confirmed by applying it to only a very simplified situation with a straight road in a rural district. By developing the above prediction method, in this report, more advanced investigation applicable to a fairly complicated acoustic environment is described by applying it to the actual road traffic noise data observed especially at a T-type intersection in a city area. At this time, the effects of the surrounding background noises are reasonably taken into account for predicting the road traffic noise.

\section{OUTLINE OF PREDICTION THEORY}

Consider the wave form of the sound level fluctuation at an observation point. Let $w\left(\xi_{i}\right)\left(\xi_{i} \triangleq t-\tau_{i}\right)$ be a normalized elementary response wave form with $w(0)=1$ on an intensity scale observed when one vehicle passes directly in front of an observation point during a specific time interval $T$. Then, it is supposed that the $i$ th vehicle generates the sound intensity $Y_{i}$ at a time $t=\tau_{i}$. In this case, 
based on the additive property of power quantities, the total sound intensity, $x(t)$, at a time $t$ is directly expressed as

$$
x(t)=\sum_{i=1}^{N} Y_{i} w\left(t-\tau_{i}\right),
$$

where $N$ denotes the number of vehicles passing directly in front of an observation point during the time interval $T$. It is quite natural to assume that the random variable $Y_{i}$ is mutually independent of the random variable $\tau_{i}$.

By regarding the number of transporting vehicles, $N$, as an arbitrary random variable and using Eq. (1) under the above conditions, one can obtain the moment statistics of $x(t)$, as follows ${ }^{1)}$ :

$$
\begin{aligned}
\langle x\rangle= & \frac{\langle N\rangle}{T}\langle Y\rangle J_{1}^{1}, \\
\left\langle x^{2}\right\rangle= & \frac{\langle N(N-1)\rangle}{T^{2}}\langle Y\rangle^{2} J_{1}^{2}+\frac{\langle N\rangle}{T}\left\langle Y^{2}\right\rangle J_{2}^{1}, \\
\left\langle x^{3}\right\rangle= & \frac{\langle N(N-1)(N-2)\rangle}{T^{3}}\langle Y\rangle^{3} J_{1}^{3} \\
& +3 \frac{\langle N(N-1)\rangle}{T^{2}}\left\langle Y^{2}\right\rangle\langle Y\rangle J_{2}^{1} J_{1}^{1} \\
& +\frac{\langle N\rangle}{T}\left\langle Y^{3}\right\rangle J_{3}^{1}, \\
\left\langle x^{4}\right\rangle= & \frac{\langle N(N-1)(N-2)(N-3)\rangle}{T^{4}}\left\langle Y^{4}\right\rangle J_{1}^{4} \\
& +6 \frac{\langle N(N-1)(N-2)\rangle}{T^{3}}\left\langle Y^{2}\right\rangle\langle Y\rangle^{2} J_{2}^{1} J_{1}^{2} \\
& +4 \frac{\langle N(N-1)\rangle}{T^{2}}\left\langle Y^{3}\right\rangle\langle Y\rangle J_{3}^{1} J_{1}^{1} \\
& +3 \frac{\langle N(N-1)\rangle}{T^{2}}\left\langle Y^{2}\right\rangle^{2} J_{2}^{2}+\frac{\langle N\rangle}{T}\left\langle Y^{4}\right\rangle J_{4}^{1}, \\
& \vdots
\end{aligned}
$$

with

$$
J_{p}^{q} \triangleq\left\{\int_{-T / 2}^{T / 2} w^{p}(\xi) d \xi\right\}^{q},
$$

where $\langle>$ denotes an averaging operation.

Based on the additive property of cumulant statistics with respect to the independent random variables, one can extend the above theory to cases when several types of vehicles pass on a multi-lane road. The above moment statistics $\left\langle x^{m}\right\rangle$ on an intensity scale can be transformed into the cumulant statistics $\kappa_{L m}$ on a decibel scale by use of the following relationship ${ }^{1)}$ :

$$
\begin{aligned}
& \frac{1}{x_{0}^{m}}\left\langle x^{m}\right\rangle=\exp \left\{\sum_{i=1}^{\infty} \frac{1}{i !}\left(\frac{m}{c}\right) \kappa_{L i}\right\} \\
& \left(x_{0}=10^{-12} \mathrm{~W} / \mathrm{m}^{2}, c \triangleq 10 / \ln 10\right) .
\end{aligned}
$$

Thus, after regarding the cumulant statistics as unknown parameters and by solving the simultaneous equations derived from Eq. (4) $(i, m=1,2$, $\ldots, M)$, the cumulant statistics $\kappa_{L i}(i=1,2, \ldots, M)$ on a decibel scale can be obtained. For example, the transformation formula for the cumulant statistics up to the fourth order is explicitly expressed by

$$
\begin{aligned}
\kappa_{L 1}= & c\left\{4 \ln \frac{\langle x\rangle}{x_{0}}-3 \ln \frac{\left\langle x^{2}\right\rangle}{x_{0}^{2}}\right. \\
& \left.+\frac{4}{3} \ln \frac{\left\langle x^{3}\right\rangle}{x_{0}^{3}}-\frac{1}{4} \ln \frac{\left\langle x^{4}\right\rangle}{x_{0}^{4}}\right\}, \\
\kappa_{L 2}= & c^{2}\left\{-\frac{26}{3} \ln \frac{\langle x\rangle}{x_{0}}+\frac{19}{2} \ln \frac{\left\langle x^{2}\right\rangle}{x_{0}^{2}}\right. \\
& \left.-\frac{14}{3} \ln \frac{\left\langle x^{3}\right\rangle}{x_{0}^{3}}+\frac{11}{12} \ln \frac{\left\langle x^{4}\right\rangle}{x_{0}^{4}}\right\}, \\
\kappa_{L 3}= & c^{3}\left\{9 \ln \frac{\langle x\rangle}{x_{0}}-12 \ln \frac{\left\langle x^{2}\right\rangle}{x_{0}^{2}}\right. \\
& \left.+7 \ln \frac{\left\langle x^{3}\right\rangle}{x_{0}^{3}}-\frac{3}{2} \ln \frac{\left\langle x^{4}\right\rangle}{x_{0}^{4}}\right\}, \\
\kappa_{L 4}= & c^{4}\left\{-4 \ln \frac{\langle x\rangle}{x_{0}}+6 \ln \frac{\left\langle x^{2}\right\rangle}{x_{0}^{2}}\right. \\
& \left.-4 \ln \frac{\left\langle x^{3}\right\rangle}{x_{0}^{3}}+\ln \frac{\left\langle x^{4}\right\rangle}{x_{0}^{4}}\right\} .
\end{aligned}
$$

In order to evaluate the $L_{x}$ sound levels (e.g., $L_{5}$, $L_{10}, L_{50}, L_{90}$ and $L_{95}$ ), the cumulative distribution function (abbr., c.d.f.) $Q(L)$ of the sound level fluctuation $L$ can be expressed in the form of a statistical Hermite series expansion type ${ }^{2)}$ universally applicable to the arbitrary non-Gaussian distribution form, as follows:

$$
\begin{aligned}
Q(L)= & \frac{1}{\sqrt{2 \pi}} \int_{-\infty}^{\left(L-\kappa_{L 1}\right) / \sqrt{\kappa_{L 2}}} \exp \left(-\zeta^{2} / 2\right) d \zeta \\
& -\sum_{n=3}^{\infty} A_{n}\left(\boldsymbol{\kappa}_{L}\right) \frac{1}{\sqrt{2 \pi}} \exp \left[-\left(L-\kappa_{L 1}\right)^{2} / 2 \kappa_{L 2}\right] \\
& \cdot H_{n-1}\left(\frac{L-\kappa_{L 1}}{\sqrt{\kappa_{L 2}}}\right)
\end{aligned}
$$

with

$$
A_{n}\left(\boldsymbol{\kappa}_{L}\right) \triangleq \frac{1}{n !}\left\langle H_{n}\left(\frac{L-\kappa_{L 1}}{\sqrt{\kappa_{L 2}}}\right)\right\rangle,
$$

where $H_{n}($.$) denotes the n$th order Hermite polynomial. 


\section{CONSIDERATION OF THE EFFECTS OF BACKGROUND NOISES}

In the actual measurement of road traffic noises, the effects of background noises can not be ignored. The following two approaches can be introduced as trials for considering the effects of background noises.

(1) The first approach is an orthodox one based on the convolution integral of the probability density function (abbr., p.d.f.), as follows ${ }^{3)}$ :

$$
Q_{\mathrm{T} 1}(L)=\int_{-\infty}^{10^{(L-120) / 10}} P_{\mathrm{T} 1}(x) d x
$$

with

$$
\boldsymbol{P}_{\mathrm{T} 1}(x)=\int_{0}^{\infty} \boldsymbol{P}_{\mathrm{R}}(x-\zeta) \boldsymbol{P}_{\mathrm{B}}(\zeta) d \zeta .
$$

Here, $P_{\mathrm{R}}(*), P_{\mathrm{B}}(*)$ and $P_{\mathrm{T}}\left({ }^{*}\right)$ are, respectively, the p.d.f. of the sound intensity fluctuation for the road traffic noise, that of the background noise and that of the objective total noise. The above equation has been derived by using the additive property of two statistically independent sound intensities.

(2) The second approach is a practical one based on paying attention to the restricted range above a certain level $L_{0}$. Provided the sound level probability distribution restricted only in the level range $\left[L_{0}, \infty\right)$ is considered, the following expression derived based on the fundamental property of a conditional probability can be employed ${ }^{4)}$ :

$$
\begin{aligned}
Q_{\mathrm{T} 2}(L)= & Q_{\mathrm{EX}}\left(L_{0}\right)+\left[1-Q_{\mathrm{EX}}\left(L_{0}\right)\right] \\
& \times\left\{Q_{\mathrm{T}}(L)-Q_{\mathrm{T}}\left(L_{0}\right)\right\} /\left\{1-Q_{\mathrm{T}}\left(L_{0}\right)\right\} .
\end{aligned}
$$

Here, $Q_{\mathrm{EX}}\left(L_{0}\right)$ denotes the experimental c.d.f. at a level value of $L_{0}$ and $Q_{\mathrm{T}}\left({ }^{*}\right)$ is defined as

$$
Q_{\mathrm{T}}(*)=\int_{-\infty}^{*} P(\zeta) d \zeta \quad\left(*=L \text { or } L_{0}\right)
$$

\section{EXPERIMENTAL WORK}

4.1 Simplification of an Elementary Response Wave Form by Use of Triangular Shape Approximation $^{5)}$

The experimental procedures can be simplified by using a triangular shape to approximate an elementary response wave form due to the surrounding sound propagation environment when one vehicle passes directly in front of an observation point during the time interval $T$. For the purpose of determining $\left\langle x^{m}\right\rangle$, it is not necessary to determine $w(\xi)$ itself accurately but is quite sufficient to obtain only a resultant value of the definite integral $\int_{-T / 2}^{T / 2} w^{m}(\xi) d \xi$ : that is, the values of the cumulant statistics on a decibel scale are not so sensitive to the exact wave form of $w(\xi)$, owing to the effects of various types of smoothing operations.

The approximation method based on a triangular shape is illustrated in Fig. 1, and described as follows:

(1) The elementary response wave form on a decibel scale is first transformed into an intensity scale with the normalized peak value.

(2) After this procedure, the transformed response wave form is approximated by use of a triangular shape so that the total sound intensity of the approximated response wave form is equal to that of the actual one.

\subsection{Predicted Results}

This prediction method has been applied to a road traffic noise data observed at a T-type road intersection in a city area. There are fairly high sound insulation barriers beside the road. The transporting vehicles have flowed only on the straight

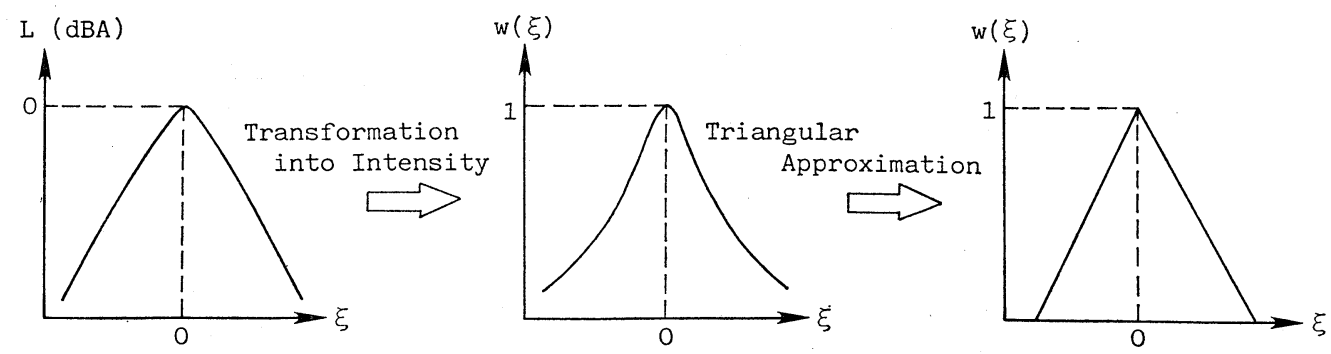

Fig. 1 Simplification procedure for the actual response wave form by use of a triangular approximation. 
road along the river. Figure 2 shows the arrangements for measuring the road traffic noise at an observation point. A photograph of the site is shown in Fig. 3. In this experiment, the passing vehicles are classified into three categories, that is, the heavy vehicles, the light vehicles or the motorcycles. The measurement period and the sampling interval for the road traffic noise have been respectively selected to $1,566 \mathrm{~s}$ and $0.5 \mathrm{~s}$ (i.e., the total number of the sampled data was 3,132). Moreover, as the data of only background noises, the data corresponding to a special case when no vehicle flows on the road have been extracted from the whole data, since these background noises expressed sufficiently the statistically stationary property within the above

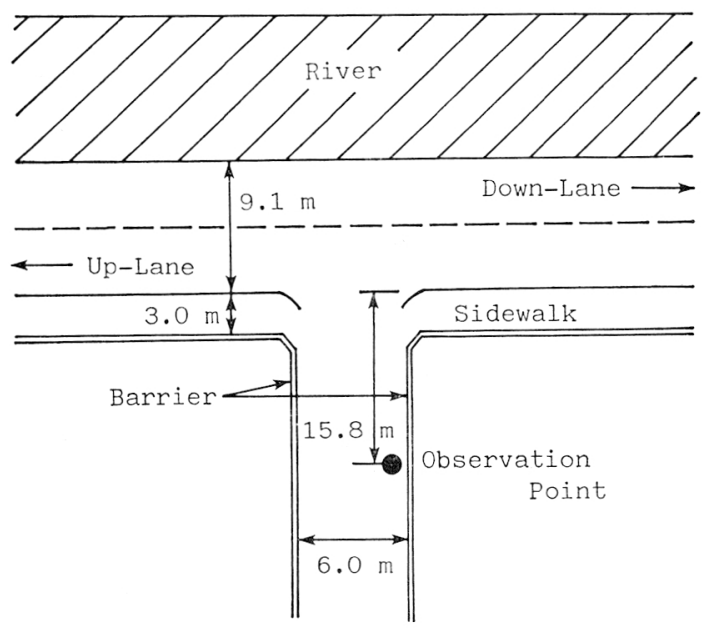

Fig. 2 Arrangements for measuring the road traffic noise at an observation point.

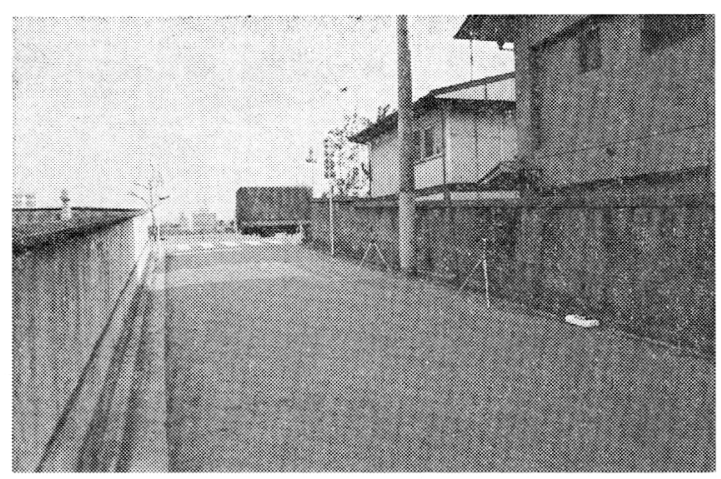

Fig. 3 The measurement site. measurement period. Table 1 shows the number of vehicles passing directly in front of an observation point during the measurement period.

Figure 4 shows a comparison between the theoretically predicted curve by use of the proposed prediction method and the experimentally sampled points for the cumulative probability distribution of the sound level fluctuation. The experimentally sampled points are marked by the circles, and the curve predicted theoretically by considering only the first and second order statistics is shown by the solid line. Therefore, the theoretically predicted curve is obtained by the first approximation of the Hermite series expansion type expression, that is, a standard Gaussian distribution. From this figure, it is obvious that the prediction accuracy is insufficient since the very important sound evaluation indices

Table 1 Number of vehicles passing directly in front of an observation point during the measurement period.

\begin{tabular}{lrc}
\hline & Up-Lane & Down-Lane \\
\hline Heavy vehicle & 4 & 13 \\
Light vehicle & 48 & 145 \\
Motorcycle & 7 & 15
\end{tabular}

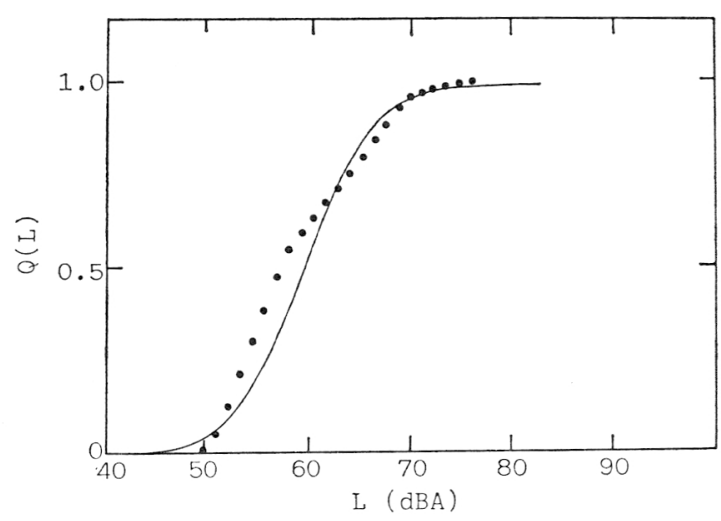

Fig. 4 A comparison between the theoretically predicted curve by use of only the lower order statistics and the experimentally sampled points for the cumulative probability distribution of the sound level fluctuation. The experimentally sampled points are marked by and the theoretically predicted curve of the first approximation of Eq. (6) (i.e., Gaussian distribution) is shown as - 


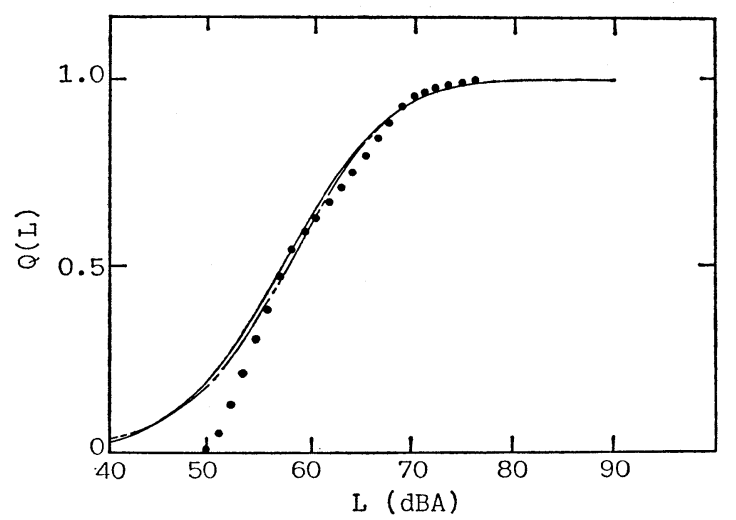

Fig. 5 A comparison between the theoretically predicted curves by use of the proposed prediction method and the experimentally sampled points for the cumulative probability distribution of the sound level fluctuation. The experimentally sampled points are marked by $\bullet$ and the theoretically predicted curves are respectively shown as —, the first approximation of Eq. (6); - - - , the second and third approximations.

such as $L_{10}$ and $L_{50}$ are not predicted so accurately. Therefore, one must consider the non-Gaussian property by use of not only the lower order statistics, but also the higher order statistics. By considering the lower and higher order statistics, a comparison between the theoretically predicted curves and the experimentally sampled points is shown in Fig. 5. Here, the solid line shows the first approximation and the dashed-dotted line shows the second and third approximations. From this figure, it is found that the theoretical curves are closer to the experimentally sampled points as the number of expansion terms increases. This is because of the consideration of the non-Gaussian property by use of the lower and higher order statistics. Furthermore, it must be noticed that the prediction error in this figure for the sound evaluation indices, $L_{5}, L_{10}$ and $L_{50}$, usually used in the evaluation and regulation problems, is within $\pm 1 \mathrm{dBA}$. However, the predicted curves in the lower level are rather insufficient, since the effect of any background noise has been not considered in this prediction.

Figure 6 shows a comparison between the theoretically predicted curve by use of the proposed prediction method and the theoretically predicted

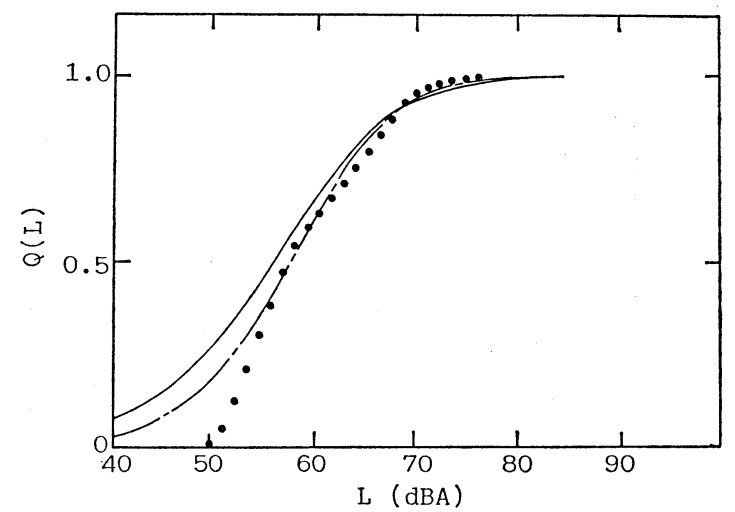

Fig. 6 A comparison between the theoretically predicted curve by use of the proposed prediction method and the theoretically predicted curve by considering only the idealized Poisson type traffic flow. The theoretically predicted curves are respectively shown as _- - , the third approximation by use of the proposed prediction method; —_, the third approximation based on the idealized Poisson type traffic flow.

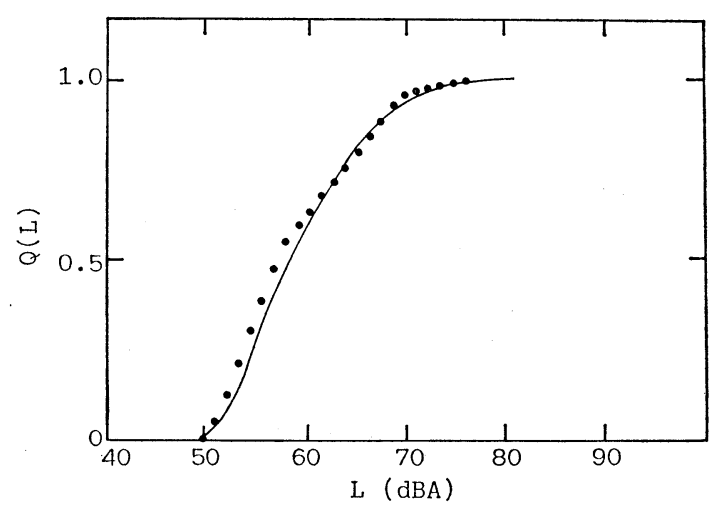

Fig. 7 A comparison between the theoretically predicted curve by use of the convolution integral and the experimentally sampled points for the cumulative probability distribution of the sound level fluctuation. The experimentally sampled points are marked by and the theoretically predicted curve by use of Eq. (8) is shown as -

curve by considering only the idealized Poisson type traffic flow. Apparently, the theoretical curve by use of the proposed method agrees well with the 


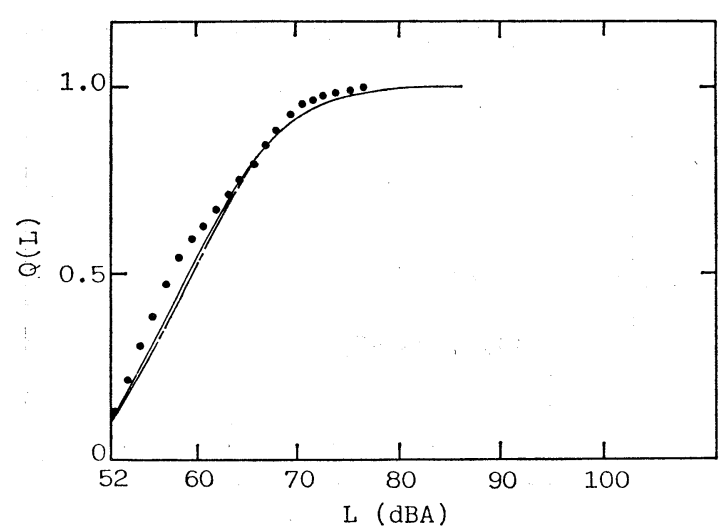

Fig. 8 A comparison between the theoretically predicted curve by use of the proposed prediction method and the experimentally sampled points for the cumulative probability distribution above the level value of $52 \mathrm{dBA}$. The experimentally sampled points are marked by 1 and the theoretically predicted curves are respectively shown as —_, the first approximation by use of Eq. (10); - - - , the second and third approximations.

experimentally sampled points, as compared with the theoretical curve by considering only the idealized Poisson type traffic flow. Thus, the proposed prediction method is generally applicable to the actual traffic flow of a non-Poisson type.

By considering the effects of background noises, Fig. 7 shows a comparison between the experimentally sampled points and the theoretically predicted curve by use of the convolution integral, Eq. (8). Moreover, Fig. 8 shows a comparison between the theoretically predicted curves by use of Eq. (10) and the experimentally sampled points for the cumulative probability distribution above $52 \mathrm{dBA}$. From these figures, it is obvious that these prediction procedures for considering the effects of background noises has a good performance for predicting a whole shape of the cumulative probability distribution under consideration.

\section{CONCLUDING REMARKS}

In this study, based on a general method of predicting a road traffic noise by use of an elementary response wave form when one vehicle passes directly in front of an observation point, its practical effectiveness has been experimentally confirmed by applying it to the actual road traffic noise observed especially at a T-type intersection.

Though this study has focused on some practical aspects, there still remain many future problems. That is, this prediction method must be applied to many other types of road intersections to broaden and confirm its further effectiveness. Next, it is necessary to establish a theoretical method for predicting quantitatively an elementary response wave form, from the methodological viewpoint. Moreover, the specific traffic flow in the arbitrary types of road intersections should be considered on the basis of this fundamental prediction method.

\section{ACKNOWLEDGEMENTS}

The authors would like to express their cordial thanks to Prof. S. Yamaguchi, Mr. F. Hirahara and Mr. K. Mikamoto for their helpful suggestions. The authors additionally acknowledge many constructive discussions in the annual meeting of the Acoustical Society of Japan. ${ }^{6)}$

\section{REFERENCES}

1) M. Ohta, A. Ikuta, and S. Kuwahara, "A unified prediction method of simple hybrid type for the road traffic noise and ground vibration for the stationary traffic flow," J. Acoust. Soc. Jpn. (J) 41, 300-307 (1985) (in Japanese).

2) M. Ohta and T. Koizumi, "General statistical treatment of the response of a linear rectifying device to a stationary random input," IEEE Trans. Inf. Theory IT-14, 595-598 (1968).

3) D. Middleton, An Introduction to Statistical Communication Theory (McGraw Hill, New York, 1960), p. 20.

4) M. Uzu, Y. Sasaki, M. Nobuso, and M. Ohta, "A practical hybrid method for prediction of road traffic noise from a viewpoint of filtered Poisson process model," J. Acoust. Soc. Jpn. (J) 39, 791-797 (1983) (in Japanese).

5) S. Yamaguchi, M. Ohta, and K. Nakamura, "A practical probabilistic prediction of road traffic noise from filtered Poisson process model with a simplified elementary time pattern of triangular type," Arch. Acoust. 10, 75-91 (1985).

6) M. Ohta and Y. Mitani, "A practical prediction method using elementary time pattern for road traffic noise (Application to T-type intersection)," Rep. Spring Meet. Acoust. Soc. Jpn., 435-436 (1987) (in Japanese). 